\title{
Información del conocimiento organizacional a través de los informes anuales publicados en las páginas web de las empresas
}

\author{
Francisca Tejedo Romero* \\ * Facultad de Ciencias Económicas y Empresariales, Universidad de Castilla-La Mancha, Albacete, España. \\ Correo-e: Francisca.Tejedo@uclm.es
}

Recibido: 18-02-2013; 2a version: 28-04-2013; Aceptado: 10-05-2013.

\begin{abstract}
Cómo citar este artículo/Citation: Tejedo Romero, F. (2014). Información del conocimiento organizacional a través de los informes anuales publicados en las páginas web de las empresas. Revista Española de Documentación Científica, 37(1):e031. doi: http://dx.doi.org/10.3989/redc.2014.1.1068

Resumen: Internet es un medio de comunicación y transmisión de información que ha dado lugar a que las empresas desarrollen su actividad en economías basadas en el conocimiento. El conocimiento se ha convertido en el recurso más importante para la creación de ventajas competitivas, en particular el conocimiento que las empresas han incorporado en sus procesos, sistemas, cultura, I+D, es decir, su conocimiento organizacional (Capital Estructural). Por ello, la publicación de información por parte de las empresas sobre su Capital Estructural puede mejorar la reputación de las mismas. Así, el objetivo de este trabajo se ha centrado en analizar la cantidad de información sobre el conocimiento organizacional que suministran las empresas en los informes anuales obtenidos de sus páginas web, así como la evolución sufrida en los años objeto de estudio.
\end{abstract}

Palabras clave: Análisis de contenido; páginas web; información; conocimiento; informe anual.

\section{Information on organizational knowledge from annual reports published on companies' websites}

Abstract: Internet is a means of communication and transmission of information that has led companies to develop their business activities in knowledge-based economies. Knowledge has become the most important resource for creating competitive advantages, specifically the knowledge incorporated by companies into their processes, systems, culture, R\&D, i.e., their organizational knowledge (Structural Capital). Therefore, Structural Capital disclosures by companies can improve their reputation. Thus, the objective of our work has focused on analysing the information on organizational knowledge supplied by companies in annual reports posted on their websites, as well as on its evolution over the years studied.

Keywords: Content analysis; web pages; information; knowledge; annual report.

Copyright: () 2014 CSIC. Este es un artículo de acceso abierto distribuido bajo los términos de la licencia Creative Commons Attribution-Non Commercial (by-nc) Spain 3.0. 


\section{INTRODUCCIÓN}

Los cambios que se han producido en la economía como consecuencia de la globalización de las actividades económicas de los negocios y, por ende, la internacionalización de los mercados, que cada vez exigen mayor transparencia, la creciente complejidad de los negocios y competitividad empresarial, al igual que el auge de las nuevas Tecnologías de la Información y de las Comunicaciones, han dado origen a una nueva realidad.

Ante este nuevo escenario, las empresas se enfrentan al reto de responder al mismo, modificando las estructuras organizativas y las formas de hacer negocio, con nuevas técnicas para gestionar las mismas (Sancho, 2007). Por ello, la capacidad de innovar de las empresas se puede considerar como uno de los principales recursos intangibles que permite generar ventajas competitivas (Carrillo-Durán y Nuño-Moral, 2010; Lascurain y otros, 2010; Ferrer, 2008; Sancho, 2007) mediante la incorporación del conocimiento en sus procesos, productos, actividades, etc., (Ortiz de Urbina, 2003), convirtiéndose éste en el eje fundamental de la innovación y del desarrollo económico de las sociedades (Bueno Campos, 1998; David y Foray, 2002; OECD, 1996).

Como consecuencia, la habilidad para adquirir información, transformarla en conocimiento, incorporarlo como aprendizaje, compartirlo rápidamente y ponerlo en práctica dónde, cómo y cuando sea necesario, constituye la capacidad organizativa más importante para enfrentarse a las turbulencias del entorno (Grant, 1996; Nonaka y Takeuchi, 1995). Es por ello que la información y el conocimiento sean considerados como las principales y grandes fuentes de ventajas competitivas (Davenport y Prusak, 1998; Ortiz de Urbina, 2003), porque estimulan el desarrollo económico, la creatividad, la invención y su aplicación, en nuevos productos, servicios y procesos, que generan la creación del valor y aumentan la productividad y rentabilidad (Drucker, 1993), y junto con el resto de recursos intangibles con que cuenta la empresa, han sido objeto de análisis de manera creciente en los últimos años.

Estos recursos presentan distintos orígenes como son las personas, la organización y el entorno socioeconómico, formando lo que se denomina "Capital Intelectual". Sveiby (1997) los clasifica en tres: competencias de empleados (o capital humano), se refiere al conocimiento que aportan las personas y que se refleja en su educación y experiencia; estructura interna (o capital estructural) que se refiere al conocimiento que procede de la organización, y que se encuentra materializado en la I+D, en la tecnología, en el estilo de gestión, en las patentes, en la cultura y en los procesos de gestión y; estructura externa (o capital relacional) que es el conocimiento que procede del exterior de la empresa, comprende relaciones con clientes y proveedores, marcas y reputación.
Además, qué duda cabe que Internet se ha convertido, para las empresas, en un canal de comunicación y de transferencia de información al exterior. De hecho, la entrada en vigor en España de la ley 26/2003, de 17 de julio, de transparencia informativa, ha obligado a las empresas que cotizan en bolsa a disponer de una página web donde suministrar información, cuyo contenido mínimo se establece en la Orden ECO/3722/2003, de 26 de diciembre.

Por tanto, conscientes de la importancia de la información y el conocimiento, el propósito de este trabajo es el estudio de aquel conocimiento que la empresa ha podido internalizar y que permanece en el tiempo dentro de la misma (Bontis, 2001), es decir, su Capital Estructural, al conseguir explicitar, sistematizar e internalizar y que, en un principio, puede estar latente en las personas y equipos de la empresa (Euroforum, 1998), quedando incluidos todos aquellos conocimientos estructurados de los que depende la eficacia y eficiencia interna de la empresa (Sveiby, 1997).

En este sentido, la publicación de información voluntaria por parte de las empresas sobre su Capital Estructural es una valiosa herramienta de información para todas las partes interesadas (stakeholders), ya que éstas desean tener mayor información acerca de los recursos que crean riqueza en las empresas, convirtiéndose en un elemento esencial de rendición de cuentas para aquellos con un interés legítimo en las mismas. El suministro de esta información puede mejorar la confianza y el compromiso de los trabajadores, dar más legitimidad a las actividades realizadas por la empresa, atraer a mejores socios y, en general, mejorar la reputación de la empresa. En consecuencia, se puede significar que la comunicación corporativa se ha convertido en una herramienta estratégica para las empresas.

No obstante, aunque a la hora de emitir información, las empresas están empleando una gran variedad de informes y de recursos (prensa especializada, folletos informativos, informes anuales, informes de capital intelectual, informes de responsabilidad social, presentaciones, conversaciones telefónicas, etc.), el informe anual es la vía que más han utilizado para suministrar información. Ello se debe a tres razones principalmente: en primer lugar, la empresa tiene el control editorial sobre el documento; en segundo lugar, es el documento más ampliamente distribuido por la empresa y, en tercer lugar, la Orden ECO/3722/2003 obliga a todas las empresas cotizadas en España a publicar en su página web el informe o memoria anual.

A este fin, el objetivo de esta investigación se ha centrado en analizar la cantidad de información sobre el conocimiento organizacional (Capital Estructural) que suministran las empresas españolas cotizadas, pertenecientes al IBEX 35, en sus informes anuales obtenidos a través de sus páginas 
web, así como la evolución sufrida en los años objeto de estudio. Para la consecución de tal objetivo se han planteado las siguientes preguntas de investigación:

- ¿Cuál es el la cantidad de información suministrada por las empresas de la muestra?

- ¿Cuál es la evolución sufrida en el nivel de información suministrado por las empresas de la muestra?

- ¿Existen patrones comunes de comportamiento entre las empresas de la muestra?

Así, y utilizando la metodología del "análisis de contenido", se ha creado un índice de divulgación del Capital Estructural, y seis subíndices correspondientes a las subcategorías del mismo, que han permitido cuantificar la cantidad de información voluntaria suministrada por las empresas.

La novedad de este estudio radica en que se realiza un análisis longitudinal con la finalidad de analizar la evolución sufrida en los niveles de información a estudiar, contribuyendo de esta manera a una mejor comprensión respecto al tratamiento de la información empresarial respecto al Capital Estructural.

A continuación, en la siguiente sección se realiza una aproximación al estado de la cuestión, posteriormente, se comentará la metodología aplicada para la realización de dicho estudio, seguida del análisis de los datos y resultados. En la quinta sección se hará alusión a las conclusiones, y se finalizará con la bibliografía.

\section{APROXIMACIÓN AL ESTADO DE LA CUES- TIÓN: ESTUDIOS DE DIVULGACIÓN}

El interés por los intangibles queda reflejado en un gran número de publicaciones y estudios, abarcando diversos ámbitos que van desde la medición y divulgación de información, la gestión y administración de empresas y el impacto en el crecimiento económico (Bueno Campos, 1998; García-Meca y Martínez, 2005; Guthrie y otros, 2006; Oliveras y otros, 2008; Sveiby, 1997; Vickery, 1999).

Sin embargo, la dificultad que plantea el hecho de identificarlos y definirlos con precisión, ha dado lugar a que no se haya normalizado ningún marco conceptual, existiendo una variedad de marcos que han sido utilizados por los investigadores para poder clasificar y definir dichos intangibles (Brooking, 1997; Euroforum, 1998; Kaplan y Norton, 1992; Sveiby, 1997).

La inmensa mayoría de los estudios de divulgación de información del capital intelectual se han basado en el marco inicial de Sveiby (1997), que posteriormente fue modificado por Guthrie y Petty (2000) en su estudio realizado en Austria y replicado y modificado por: Brennan (2001) en Irlanda; April y otros (2003) en Suráfrica; Bozzolan y otros
(2003) en Italia; Goh y Lim (2004) en Malasia; Abeysekera y Guthrie (2005) en Sri Lanka; Vandemaele y otros (2005) en Suecia, Reino Unido y Países Bajos; Guthrie y otros (2006) en Hong Kong y Australia; Ensslin y Carvalho (2007) en Brasil; Steenkamp (2007) en Nueva Zelanda; Gan y otros (2008) en Malasia; Oliveras y otros (2008) en España, entre otros.

Estas investigaciones analizan el informe anual de las empresas cotizadas, y estudian cuál de las categorías del capital intelectual (humano, relacional y estructural) ha sido la más divulgada. Son trabajos descriptivos y transversales que consideran un ejercicio económico. Sin embargo, son escasos los estudios longitudinales que pretenden analizar la evolución experimentada en la cantidad de información suministrada durante un periodo de tiempo.

En este sentido, Abeysekera y Guthrie (2005) examinan los informes bianuales de 30 empresas listadas en Colombo Stock Exchange (principal bolsa de valores en Sri Lanka) en 1998/1999 y 1999/2000, y encuentran un incremento en el suministro de la información divulgada en dicho periodo, siendo la categoría más divulgada la correspondiente al capital relacional, seguida del humano y del estructural.

Vandemaele y otros (2005) estudian los informes anuales en 1998, 2000,y 2002, de 60 empresas suecas, de Reino Unido y de los Países Bajos. De Suecia, eligen 20 empresas que cotizan en el índice OMX. De Reino Unido, se seleccionan 20 empresas que cotizan en el índice FTSE100. Por último, para los Países Bajos escogen 20 empresas listadas en el índice AEX. Observan que se produce un aumento en el nivel de información divulgado para el periodo, siendo, en promedio, las empresas suecas las que más revelan respecto de los Países Bajos y del Reino Unido; aunque para las empresas suecas se produce un ligero retroceso entre los años 2000 y 2002 . El $40 \%$ informa sobre el capital relacional, un $30 \%$ del estructural y otro $30 \%$ del humano.

Por su parte, Guthrie y otros (2006) analizan 20 informes anuales de empresas australianas para el año 1998, y 50 informes de empresas de Australia y 100 de Hong Kong para el año 2002. Las empresas se encuentran cotizadas en la Bolsa de Valores de Australia (ASX) y en la de Hong Kong, respectivamente. Las australianas en 2002 revelan una notable mayor cantidad de información en comparación con las empresas australianas en 1998 y con las de Hong Kong para 2002. Además, existe un cambio significativo en los patrones de las australianas en 2002 , divulgándose un $49 \%$ de capital relacional, un $41 \%$ del estructural y un $10 \%$ del humano.

En España, Oliveras y otros (2008) analizan los informes anuales de 12 de las principales empresas españolas, incluidas en el IBEX 35, desde el año 2000 hasta 2002. Durante el período se ha 
incrementado el volumen de información, siendo el área más divulgada la relacionada con el capital relacional $(59,6 \%)$, seguida del humano $(21,9 \%)$ y del estructural $(18,5 \%)$.

Por otro lado, estas investigaciones se centran en empresas de gran tamaño, con una alta capitalización bursátil (muchas de ellas pertenecientes a índices selectivos de cotización), ya que, atendiendo a los postulados de la Teoría de los interesados o stakeholders (Freeman, 1984), son las grandes empresas las que tienden a tener un mayor número de interesados en la información voluntaria que divulgan, puesto que están más expuestas a la opinión pública. Además, la supervivencia y éxito a largo plazo de la empresa se encuentran sometidos a la aprobación de los grupos de interés. Por ello, las empresas están interesadas en suministrar información voluntaria acerca de los recursos intangibles que crean riqueza a largo plazo, a fin de aumentar el valor percibido de la misma por las partes interesadas (Abeysekera y Guthrie, 2005).

En este sentido, la aportación de esta investigación reside en analizar, durante un periodo más amplio, en concreto de cinco años, un total de 115 informes anuales correspondientes a 23 empresas del IBEX 35. Para ello, se estudia, exclusivamente el Capital Estructural, al considerar que es la categoría más importante del capital intelectual, por ser el conocimiento organizacional que permanece siempre en la empresa. Además, se realiza un examen exhaustivo sobre los elementos intangibles que lo configuran.

\section{METODOLOGÍA}

El análisis planteado se ha realizado mediante el empleo de la metodología del análisis de contenido (Chaín, 2005; Ferran-Ferrer y Pérez-Montoro, 2009; Ramos y Clabo, 2008), bajo un enfoque de tipo cuantitativo (al hacer hincapié en la cuantificación de la recogida y análisis de los datos), adoptando un método deductivo (Holsti, 1969).

Para garantizar la fiabilidad del análisis de contenido se han considerado las indicaciones de Guthrie y otros (2004) que consideran que, para el caso de un único codificador si éste ha sido objeto de un período suficiente de formación y las decisiones de codificación han llegado a un nivel aceptable en la muestra piloto realizada, se puede probar la fiabilidad en el proceso de depuración de los datos. No obstante, para suplir la falta de transparencia en la codificación de los datos (Beattie y Thomson, 2007), se han llevado a cabo las etapas que se desarrollan a continuación:

\section{a) Periodo de estudio}

El periodo elegido abarca 5 años, desde el 2004 al 2008 (ambos inclusive). Esta elección viene motivada tras la aprobación en España de la ley $26 / 2003$, de 17 de julio, de transparencia informativa, que regula a todas las sociedades emisoras de valores e instrumentos financieros admitidos a cotización, donde se recoge, en el apartado 2 del artículo 117, que dichas sociedades deben disponer de una página web para difundir información, lo que facilita el acceso, a partir de 2004, a los documentos analizados de forma más fácil y accesible, dejándose para posteriores trabajos el periodo 20082012, a raíz de una serie de fusiones de empresas que hubieran distorsionado la serie de datos.

\section{b) Definición de la población y elección de la muestra}

Las empresas cotizadas en el mercado continuo español que estuviesen incluidas en el índice bursátil del IBEX 35 en el año 2008 constituyen la población de partida, al ser éstas más susceptibles de una mayor transparencia informativa.

Se opta por un diseño de la muestra dirigido o no probabilístico (Hernández y otros, 2006), considerando las empresas incluidas en el índice en el año 2008 (puesto que en años posteriores han tenido lugar operaciones de fusiones y algunas empresas han salido de dicho índice) y manteniéndolas fijas durante el resto de años, hasta el 2004. La muestra queda configurada por 23 empresas, lo que representa un $65,7 \%$ de la población de partida. Además, se encuentran caracterizados todos los sectores industriales (ver tabla I).

Tabla I. Porcentaje de participación de los sectores

\begin{tabular}{lcccc}
\hline \multirow{2}{*}{ Sectores } & \multicolumn{2}{c}{ Población inicial } & \multicolumn{2}{c}{ Muestra final } \\
\cline { 2 - 5 } & Empresas & Porcentaje & Empresas & Porcentaje \\
\hline Bienes de consumo & 2 & $5,71 \%$ & 1 & $4,35 \%$ \\
\hline Materiales básicos/Industria y construcción & 11 & $31,43 \%$ & 6 & $26,09 \%$ \\
\hline Petróleo y energía & 8 & $22,86 \%$ & 7 & $30,43 \%$ \\
\hline Servicios de consumo & 3 & $8,57 \%$ & 2 & $8,70 \%$ \\
\hline Servicios financieros e inmobiliarios & 9 & $25,71 \%$ & 5 & $21,74 \%$ \\
\hline Tecnología y telecomunicaciones & 2 & $5,71 \%$ & 2 & $8,70 \%$ \\
\hline TOTAL & $\mathbf{3 5}$ & $\mathbf{1 0 0} \%$ & $\mathbf{2 3}$ & $\mathbf{1 0 0} \%$ \\
\hline
\end{tabular}


Por otro lado, y como ya se ha comentado anteriormente, son empresas de gran tamaño. Con una media de empleados de 45.478 personas y un valor medio de capitalización bursátil que asciende a 18. 033 millones de euros (ver tabla II). Los datos y la información obtenida son referidos a nivel de grupo de empresarial.

\section{c) Selección y definición de las unidades de análisis}

La selección de las unidades de análisis más adecuadas es una cuestión importante en la recopilación de datos, ya que se pretende codificar información en distintas categorías. Debe quedar claro qué datos se analizan, de qué manera se definen y de qué documentos se extraen.

Así, las unidades de muestreo han sido los informes anuales, las de registro son la presencia o ausencia de información y en las unidades contexto se ha analizado la información a nivel de frase.

\section{d) Adopción de un marco para clasificar la in- formación}

Para la codificación de los datos se ha seguido el marco de Sveiby (1997) y las modificaciones que se han realizado en el mismo (Guthrie y Petty, 2000; Abeysekera y Guthrie, 2005, Guthrie y otros, 2006; Abeysekera, 2007; Steenkamp, 2007; Gan y otros, 2008). El modelo de Capital Estructural está formado por 6 subcategorías.

Así y, con la finalidad de que el proceso de codificación sea transparente y claro, para poder realizar el proceso de codificación de los datos, se ha procedido a definir cada una de las categorías y elementos del modelo en base a la literatura previa (Guthrie y Petty, 2000; Abeysekera y Guthrie, 2005; Guthrie y otros, 2006; Abeysekera, 2007; Steenkamp, 2007; Gan y otros, 2008; Li y otros, 2008), quedando formado por 6 subcategorías y un total de 12 elementos que tienen carácter intangible, es decir, naturaleza no física:

PROPIEDAD INTELECTUAL: Son aquellos conocimientos que la empresa ha protegido legalmente mediante su inscripción en los registros correspondientes.
1. Patentes: Es un derecho de propiedad exclusivo que concede el Estado a su inventor por un tiempo limitado que excluye a otros a copiar, fabricar o vender la invención durante ese período de tiempo (Li y otros, 2008; Steenkamp, 2007).

2. Copyrights: Es el derecho a explotar un trabajo o una obra. Al igual que las marcas, pueden o no pueden ser legalmente protegidos (Li y otros, 2008).

3. Marcas comerciales: Se pueden registrar o no. Las marcas registradas pueden ser un nombre, logotipo, una imagen o una combinación, y también puede ser utilizada para asociarla con la empresa o sus productos. No sólo permite la identificación de bienes y servicios sino también representa el prestigio de sus fabricantes ( $\mathrm{Li}$ y otros, 2008; Steenkamp, 2007).

4. Secretos comerciales: Son una alternativa a las patentes, aunque éstos sólo son viables si la tecnología se puede mantener como un secreto después de dar a conocer la producción al público (Li y otros, 2008).

5. FILOSOFÍA DE GESTIÓN: Es la visión del negocio o actividad que lleva a cabo la empresa. Por tanto, es la forma que los líderes tienen de entender y gestionar su negocio. Se hace hincapié en la presencia de una línea de actuación directiva en materia de gestión respecto a empleados, clientes, medioambiente y comunidad. Algunos indicadores pueden hacer referencia a: la creación de valor para los accionistas, el crecimiento sostenible, dialogo con los stakeholders, proteger el medioambiente y cuidar de la sociedad (Abeysekera, 2007; Abeysekera y Guthrie, 2005; Gan y otros, 2008; Li y otros, 2008; Steenkamp, 2007).

6. CULTURA CORPORATIVA: Es el conjunto de valores, normas y formas de actuar que son reconocidos y compartidos por los miembros de una empresa, condicionando su comportamiento y los resultados corporativos. Es decir, como indicadores pueden hacer referencia a: la misión, códigos éticos, código de conducta, código de práctica y los principios de funcionamiento (Abeysekera, 2007; Abeysekera y Guthrie, 2005; Gan y otros, 2008; Li y otros, 2008; Steenkamp, 2007).

Tabla II. Estadísticos descriptivos del tamaño de las empresas de la muestra

\begin{tabular}{lrr}
\hline Tamaño & Mínimo & Máximo \\
\hline Capitalización bursátil (millones C) & 1.859 & 106.067 \\
\hline No empleados & 891 & 251.775 \\
\hline
\end{tabular}


PROCESOS TECNOLÓGICOS Y DE GESTIÓN: Se refieren a los mecanismos que implementan la filosofía de gestión de la empresa, incluyendo: sistemas, políticas, procedimientos, reingeniería y otros procesos y certificaciones de calidad asociados con la empresa (Brooking, 1997; Guthrie y otros, 2006).

7. Procesos de Gestión: No basta con tener una cultura corporativa y una filosofía de gestión, es necesario establecer mecanismos para poner en práctica la filosofía empresarial y asegurar que cada cual ocupe el puesto más adecuado para que las cosas salgan de la mejor manera posible. La tipología de los procesos que deben poner en marcha los gerentes es variada, entre las que destacan las políticas y procesos de control de calidad (Abeysekera, 2007; Abeysekera y Guthrie, 2005; Gan y otros, 2008; Li y otros, 2008; Steenkamp, 2007).

8. Procesos Tecnológicos: Cualquier actividad realizada dentro de la empresa que contribuya a la creación de capital estructural (Abeysekera, 2007; Abeysekera y Guthrie, 2005; Li y otros, 2008; Steenkamp, 2007).

$\boldsymbol{I}+\boldsymbol{D}+\boldsymbol{i}$ : Es el esfuerzo realizado por la empresa consistente en la realización de determinadas actividades en I+D junto con aquellas que aportan un grado de novedad significativo con respecto a la posición tradicional de la empresa en el mercado.

9. Investigación y Desarrollo: Son aquellas actividades llevadas a cabo por la empresa que permiten alcanzar unos niveles superiores de conocimiento y la aplicación de éstos en la mejora de la actividad empresarial, permitiendo explotar una ventaja competitiva. Incluye, por ejemplo, políticas y programas de $I+D$, número de proyectos emprendidos por la empresa, tasa de éxito de los proyectos, etc. (Li y otros, 2008).

10. Innovación: Es la implementación exitosa de ideas creativas dentro de la empresa mediante la introducción de algo nuevo y útil en los productos, procesos o servicios (Li y otros, 2008).

SISTEMAS DE INFORMACIÓN Y DE REDES: Son los sistemas integrados de redes de todos los canales de comunicación utilizados en la empresa.

11. Sistemas de información: Son los manuales y la tecnología basada en sistemas para mantener la gestión, compartir y difundir información, así como la red de personas, con el fin de obtener acceso a la información. Los sistemas de información proporcionan los medios para implementar los procesos de gestión, tales como base de datos, red informática, hardware, software, etc. (Abeysekera, 2007; Abeysekera y Guthrie, 2005; Gan y otros, 2008; Steenkamp, 2007).

12. Sistemas de redes: Los sistemas de red son sistemas de información que tienen la capacidad de conectarse con otros sistemas con el fin de obtener acceso a los proveedores y clientes y a la información de otras bases de datos. Por ejemplo, internet, videoconferencia, fax, etc. (Abeysekera, 2007; Abeysekera y Guthrie, 2005; Gan y otros, 2008; Li y otros, 2008; Steenkamp, 2007).

\section{e) Establecimiento del sistema de cuantifica- ción mediante las reglas de recuento}

La cuantificación de las unidades de registro se ha realizado mediante el análisis de la presencia/ ausencia (Krippendorff, 2004) de los distintos elementos intangibles.

En este sentido, para el estudio de las 6 subcategorías de Capital Estructural se han utilizado índices no ponderados con la finalidad de agrupar los elementos intangibles en sus subcategorías para poder cuantificar el nivel de información suministrado:

$$
\begin{aligned}
& \text { I Propiedad Intelectual }_{j}=\frac{1}{4} \sum_{i=1}^{4} X_{i j} \\
& \text { I Filosofia Gestión }_{j}=\frac{1}{1} \sum_{i=1}^{1} X_{i j} \\
& \text { I Cultura Corporativa }_{j}=\frac{1}{1} \sum_{i=1}^{1} X_{i j}
\end{aligned}
$$$$
\text { I Procesos Tecnológicos y Gestión } n_{j}=\frac{1}{2} \sum_{i=1}^{2} X_{i j}
$$$$
I I+D+i_{j}=\frac{1}{2} \sum_{i=1}^{2} X_{i j}
$$

I Sistemas Información Redes ${ }_{j}=\frac{1}{2} \sum_{i=1}^{2} X_{i j}$

Donde $I_{j}$ es el índice de divulgación no ponderado de la empresa $j, i$ el elemento intangible, $X_{i j}$ es la puntuación obtenida por la empresa j del elemento $i$. Consecuentemente, $X_{i j}$ tomará el valor de 1 si la empresa $j$ ha divulgado el elemento intangible $i \mathrm{y}$, en caso contrario, tomará el valor de 0 si no ha sido divulgado.

Además, se ha obtenido un índice de divulgación del Capital Estructural (I Capital Estructural) para la empresa $j$, en la misma línea que los anteriores, que será la agregación de los mismos, o bien, la agregación de todos los elementos intangibles, que en concreto ascienden a 12 .

$$
\text { I Capital Estructural }{ }_{j}=\frac{1}{12} \sum_{i=1}^{12} X_{i j}
$$


Se ha codificado tanto la información cuantitativa como cualitativa. No se realiza un recuento de frecuencias de los elementos, es decir, no se consideran las repeticiones. Así, a modo de ejemplo encontramos que:

"El Grupo ACERINOX persigue: asegurar un crecimiento rentable y sostenido de su negocio remunerar a sus accionistas a través del valor de la acción y el reparto de dividendos; mantenerse a la cabeza de su sector, incrementando su presencia internacional y la implantación de sus productos; Ofrecer seguridad y desarrollo profesional a sus empleados, aportando, paralelamente, mayores cotas de bienestar a los ciudadanos; contribuir al mantenimiento y a la mejora del medio ambiente; contribuir al desarrollo y bienestar de las comunidades en las que lleva a cabo su actividad" (Informe Anual de Acerinox 2007, p. 53).

La empresa Acerinox está describiendo de manera cualitativa la visión de su negocio, es decir, hace referencia a la Filosofía de gestión. Por tanto, en la hoja de codificación de la empresa Acerinox para el ejercicio 2007, figurará un 1 en el elemento, Filosofía de gestión.

".......... y más de 14 patentes relacionadas con las tecnologías de desalinización, los reactores biológicos de membranas, y la reutilización, avalan el éxito de la investigación aplicada por la Compañía" (Informe Anual de Acciona, 2008, p. 63).

En el párrafo anterior, Acciona informa sobre sus patentes, tanto de manera cuantitativa como cualitativa. Así, para el ejercicio 2008 en la hoja de codificación de la empresa Acciona, y en el elemento correspondiente a patentes, se codificará con una puntuación de 1 .

Tras realizar la codificación de los datos, y con el objetivo de poder resolver las preguntas de investigación planteadas, se ha llevado a cabo el tratamiento estadístico de los datos.

En primer lugar, se ha realizado un análisis descriptivo con la finalidad de explorar si los elementos intangibles, que conforman el Capital Estructural, son comunicados por las empresas de la muestra durante los años objeto de estudio. Además, y para conocer el comportamiento de las variables, se han obtenido las principales medidas de tendencia, dispersión y distribución. Mediante esta técnica se obtiene un conocimiento básico de los datos, así como la importancia de cada una de las variables evaluadas.

En segundo lugar, y para conocer si se ha modificado el nivel de divulgación de la información a lo largo del tiempo, se utiliza el test no paramétrico de Friedman para contrastar la posible existencia de diferencias significativas en la media de los rangos de las variables analizadas. También se utiliza el coeficiente de concordancia $W$ de Kendall para medir el grado de relación entre los distintos ejercicios económicos analizados, que se basa en los mismos rangos asignados en la prueba de Friedman. Si dicha prueba resultase significativa, para un p-valor $<0,05$, sería necesario realizar pruebas post hoc, en concreto, la prueba de los rangos con signo de Wilcoxon para conocer entre qué pares de años resultan significativas dichas diferencias.

Por último, para resolver la existencia de patrones de comportamiento en las empresas de la muestra, se utiliza la prueba no paramétrica de Kruskall-Wallis. Si dicha prueba resultase significativa ( $p$-valor $<0,05$ ), sería necesario realizar pruebas post hoc; en concreto, el test de DunnBonferroni para conocer entre qué pares de sectores resultan significativas dichas diferencias.

Se utilizan test no paramétricos ante la falta de normalidad en los datos, la cual queda justificada si se tiene en cuenta que, según el Teorema Central del Límite, una de las condiciones para el cumplimiento de normalidad de las variables es que el número de individuos de la muestra sea superior a 30.

Para la realización del análisis de contenido se ha utilizado el programa MAXQDA, aunque la codificación se ha realizado de manera manual, y las técnicas estadísticas empleadas se han realizado mediante el programa SPSS.

\section{ANÁLISIS DE LOS DATOS Y RESULTADOS}

En la tabla III se observa que en el año 2004, 2005 y 2006 la filosofía de gestión ha sido el elemento más divulgado, un total de 20 empresas lo han revelado, lo que representa un $86,96 \%$ de la muestra. En este sentido, las empresas están haciendo especial hincapié en proyectar la visión de su negocio de cara a que los grupos de interés tengan conocimiento de ello y lo valoren de manera positiva. Para el año 2007 el elemento más divulgado ha sido el referente a sistemas de redes, procesos de gestión y cultura corporativa que ha sido revelado por el $82,61 \%$ de empresas de la muestra, es decir, por 19 empresas. Para el 2008 se incrementa en una empresa más que suministra información respecto del sistema de redes y los procesos de gestión con un $86,96 \%$ de empresas que lo divulgan.

Sin embargo, para el periodo objeto de estudio, los elementos menos divulgados han sido los secretos comerciales, quizás por el miedo que tienen las empresas a su competencia, ya que estos secretos suponen una gran ventaja competitiva y no desean hacerlos públicos puesto que pueden ser imitados por las empresas de su sector. También, prácticamente ninguna empresa suministra información relativa a los copyrights, posiblemente porque no disponen de ellos.

A continuación, de la tabla IV se desprende que para el período de análisis, la mayoría de las empresas suministran información relativa a la filosofía de su organización, con un valor medio en el índice de divulgación que asciende a 0,84; así, independien- 
temente del sector de pertenencia de las empresas, es lógico que éstas hagan referencia a la línea de actuación llevada a cabo por la misma (su visión) en el informe anual, en aras a obtener la máxima creación de valor para sus grupos de interés, ya que el informe va dirigido a estos grupos o stakeholders.

En este sentido, ocurre lo mismo con el índice de la subcategoría de cultura corporativa. Sin embargo, son las empresas pertenecientes al sector de Bienes de Consumo las que menos inciden en divulgar su manera de actuar, quizás es una manera de evitar una regulación más restrictiva en este sector y prefieren desviar la atención de los grupos de interés hacia otros intangibles menos conflictivos.

Por último, señalar que la subcategoría referente a la propiedad intelectual ha sido la que presenta un menor valor en su subíndice, siendo las empresas pertenecientes al sector de las Tecnologías de las Comunicaciones las que más información al respecto presenta, cuestión lógica ya que es el sector más intensivo en I+D+i, y muchos de los resultados de sus proyectos que han resultado exitosos, desean protegerlos legalmente.

Finalmente, se ha obtenido el índice de divulgación del Capital Estructural (ver tabla V), donde se refleja que para el período 2004-2005, prácticamente, la mitad de las empresas suministran información sobre su Capital Estructural, más concretamente un 49\%. Además el índice se encuentra cercano al valor medio, ya que la dispersión, medido a través de la desviación típica, no es muy elevada, en torno a un 0,23.

Tabla III. Número y porcentaje de empresas que comunican los elementos intangibles de Capital Estructural

\begin{tabular}{|c|c|c|c|c|c|c|c|c|c|c|}
\hline \multirow{3}{*}{ ELEMENTOS INTANGIBLES } & \multicolumn{10}{|c|}{ AÑos } \\
\hline & \multicolumn{2}{|c|}{2004} & \multicolumn{2}{|c|}{2005} & \multicolumn{2}{|c|}{2006} & \multicolumn{2}{|c|}{2007} & \multicolumn{2}{|c|}{2008} \\
\hline & $\mathbf{n}$ & $\%$ & $\mathbf{n}$ & $\%$ & $\mathbf{n}$ & $\%$ & $\mathbf{n}$ & $\%$ & $\mathbf{n}$ & $\%$ \\
\hline Sistemas de redes & 17 & 73,91 & 17 & 73,91 & 18 & 78,26 & 19 & 82,61 & 20 & 86,96 \\
\hline Sistemas de información & 11 & 47,83 & 13 & 56,52 & 16 & 69,57 & 13 & 56,52 & 13 & 56,52 \\
\hline Innovación & 16 & 69,57 & 15 & 65,22 & 14 & 60,87 & 15 & 65,22 & 16 & 69,57 \\
\hline Investigación y Desarrollo & 16 & 69,57 & 13 & 56,52 & 16 & 69,57 & 17 & 73,91 & 17 & 73,91 \\
\hline Procesos Tecnológicos & 12 & 52,17 & 14 & 60,87 & 12 & 52,17 & 11 & 47,83 & 9 & 39,13 \\
\hline Procesos de Gestión & 19 & 82,61 & 19 & 82,61 & 19 & 82,61 & 19 & 82,61 & 20 & 86,96 \\
\hline Cultura Corporativa & 18 & 78,26 & 17 & 73,91 & 17 & 73,91 & 19 & 82,61 & 16 & 69,57 \\
\hline Filosofía de Gestión & 20 & 86,96 & 20 & 86,96 & 20 & 86,96 & 18 & 78,26 & 19 & 82,61 \\
\hline Secretos comerciales & 1 & 4,35 & 0 & 0,00 & 0 & 0,00 & 0 & 0,00 & 0 & 0,00 \\
\hline Marcas comerciales & 1 & 4,35 & 1 & 4,35 & 4 & 17,39 & 2 & 8,70 & 0 & 0,00 \\
\hline Copyrights & 1 & 4,35 & 0 & 0,00 & 0 & 0,00 & 0 & 0,00 & 0 & 0,00 \\
\hline Patentes & 2 & 8,70 & 3 & 13,04 & 2 & 8,70 & 3 & 13,04 & 2 & 8,70 \\
\hline
\end{tabular}

Tabla IV. Media de los índices de divulgación de las distintas subcategorías

\begin{tabular}{lcccccc}
\hline \multirow{2}{*}{ SUBCATEGORÍAs } & \multicolumn{5}{c}{ Media } \\
\cline { 2 - 7 } & $\mathbf{2 0 0 4}$ & $\mathbf{2 0 0 5}$ & $\mathbf{2 0 0 6}$ & $\mathbf{2 0 0 7}$ & $\mathbf{2 0 0 8}$ & $\mathbf{2 0 0 4 - 2 0 0 8}$ \\
\hline Índice Sistemas de Información y de Redes & 0,61 & 0,65 & 0,74 & 0,70 & 0,70 & $\mathbf{0 , 6 8}$ \\
\hline Índice I+D+i & 0,70 & 0,61 & 0,65 & 0,70 & 0,70 & $\mathbf{0 , 6 7}$ \\
\hline Índice Procesos Tecnológicos y de Gestión & 0,67 & 0,72 & 0,67 & 0,65 & 0,65 & $\mathbf{0 , 6 7}$ \\
\hline Índice Cultura Corporativa & 0,78 & 0,74 & 0,74 & 0,83 & 0,83 & $\mathbf{0 , 7 6}$ \\
\hline Índice Filosofía de Gestión & 0,87 & 0,87 & 0,87 & 0,78 & 0,78 & $\mathbf{0 , 8 4}$ \\
\hline Índice Propiedad Intelectual & 0,05 & 0,04 & 0,07 & 0,05 & 0,05 & $\mathbf{0 , 0 5}$ \\
\hline
\end{tabular}

Tabla V. Media y desviación típica del índice de divulgación del Capital Estructural

\begin{tabular}{lll}
\hline Índice de Capital Estructural & Media & Desviación típica \\
\hline $\mathbf{2 0 0 4}$ & 0,49 & 0,25 \\
\hline $\mathbf{2 0 0 5}$ & 0,48 & 0,23 \\
\hline $\mathbf{2 0 0 6}$ & 0,50 & 0,25 \\
\hline $\mathbf{2 0 0 7}$ & 0,49 & 0,24 \\
\hline $\mathbf{2 0 0 8}$ & $\mathbf{0 , 4 8}$ & 0,22 \\
\hline $\mathbf{2 0 0 4 - 2 0 0 8}$ & $\mathbf{0 , 4 9}$ & $\mathbf{0 , 2 3}$ \\
\hline
\end{tabular}


Estos resultados se deben comparar con extrema cautela, puesto que los trabajos previos han considerado países y periodos de análisis diferentes, e incluso la cantidad de elementos que forman los índices y su construcción es distinta, ya que la finalidad era reflejar la importancia de cada una de las categorías del capital intelectual (humano, relacional y estructural) sobre el total. Sin embargo, en algunos de los trabajos se han podido extrapolar los resultados debido a la transparencia en el proceso de realización del análisis de contenido. La investigación de Guthrie y Petty (2000) se centra en una muestra de 20 empresas en 1998 y obtienen una puntuación en el índice de divulgación para el Capital Estructural de 53 puntos respecto de los 9 elementos intangibles que lo forman, por tanto, un 30\% de la muestra suministra información. Este estudio que es replicado por Brennan (2001) en 11 empresas irlandesas para 1999, tan sólo obtiene una puntuación en el índice de 12 puntos, lo que refleja que sólo un $12 \%$ de las empresas irlandesas revelan información. Por su parte, Goh y Lim (2004), encuentran unos resultados más cercanos a los de este trabajo, para una muestra de 20 empresas de Malasia en el año 2001, logran una puntuación de 107 puntos para los 9 elementos intangibles, lo que representa que el $59 \%$ de la muestra divulga información.

Ahora bien, en la tabla VI se recogen los resultados de la Prueba de Friedman y $W$ de Kendall, con la finalidad de conocer si los cambios que ha habido en los distintos años han resultado significativos.
Estos rangos indican que los ejercicios 2006 y 2007 presentan, por este orden, los mayores niveles de divulgación de información del Capital Estructural. Si se observa el estadístico de contraste $\chi^{2}(4)=1,813 \mathrm{su}$ p-valor es superior a 0,05 , no se podrá rechazar la hipótesis nula. Por tanto, dado que las diferencias observadas entre las 5 medidas de rangos no son estadísticamente sigsnificativas, según la prueba de Friedman, se puede afirmar que el nivel de divulgación del Capital Estructural durante los distintos ejercicios económicos analizados no varía significativamente.

Además, con el coeficiente de concordancia de Kendall se comprueba si " $k$ " muestras relacionadas proceden de la misma población. Así, cuanto más iguales sean los rangos promedio, menor concordancia existirá. Esta prueba se puede interpretar como una medida de acuerdo, varía de 0 (sin acuerdo) a 1 (con acuerdo). En este caso, el coeficiente $W$ de Kendall es igual a 0,020, que, al ser próximo a cero, indica una falta de concordancia en el nivel de divulgación de la información sobre Capital Estructural entre los distintos ejercicios económicos analizados.

Por tanto, se puede afirmar que, para el período objeto de estudio 2004-2008, la cantidad de información suministrada por las empresas, relativa a su Capital Estructural, no se ha visto modificada significativamente para cada uno de los años.

A continuación, en la tabla VII se trata de analizar si han existido diferencias significativas en la cantidad de información suministrada en función del sector de pertenencia de las empresas.

Tabla VI. Prueba de Friedman y W de Kendall para el índice de Capital Estructural

\begin{tabular}{|c|c|c|c|}
\hline \multicolumn{4}{|c|}{ Prueba de Friedman } \\
\hline Rangos & \multicolumn{3}{|c|}{ Estadísticos de contraste $^{a}$} \\
\hline & \multicolumn{3}{|c|}{ Rango promedio } \\
\hline Índice Capital Estructural 2004 & 2,98 & $\mathrm{~N}$ & 23 \\
\hline Índice Capital Estructural 2005 & 2,80 & Chi-cuadrado & 1,813 \\
\hline Índice Capital Estructural 2006 & 3,26 & $\mathrm{gl}$ & 4 \\
\hline Índice Capital Estructural 2007 & 3,11 & Sig. asintót. & 0,770 \\
\hline Índice Capital Estructural 2008 & 2,85 & & \\
\hline \multicolumn{4}{|c|}{ Prueba W de Kendall } \\
\hline Rangos & \multicolumn{3}{|c|}{ Estadísticos de contraste } \\
\hline & \multicolumn{3}{|c|}{ Rango promedio } \\
\hline Índice Capital Estructural 2004 & 2,98 & $\mathrm{~N}$ & 23 \\
\hline Índice Capital Estructural 2005 & 2,80 & W de Kendall ${ }^{\mathrm{b}}$ & 0,020 \\
\hline Índice Capital Estructural 2006 & 3,26 & Chi-cuadrado & 1,813 \\
\hline Índice Capital Estructural 2007 & 3,11 & $\mathrm{gl}$ & 4 \\
\hline Índice Capital Estructural 2008 & 2,85 & Sig. asintót. & 0,770 \\
\hline
\end{tabular}

Tabla VII. Test de Kruskall-Wallis para la hipótesis 2

\begin{tabular}{|c|c|c|c|c|c|}
\hline \multicolumn{2}{|c|}{ SECTORES } & \multirow{2}{*}{ Número } & \multirow{2}{*}{$\begin{array}{c}\begin{array}{c}\text { Rango } \\
\text { promedio }\end{array} \\
40,70\end{array}$} & \multirow[t]{2}{*}{$\begin{array}{c}\text { Chi- } \\
\text { cuadrado }\end{array}$} & \multirow[t]{2}{*}{$\begin{array}{c}\text { Sig. } \\
\text { asintót. }\end{array}$} \\
\hline \multirow{6}{*}{ 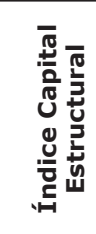 } & Bienes de Consumo & & & & \\
\hline & Materiales básicos/Industria y Construcción & 30 & 66,23 & \multirow{5}{*}{15,362} & \multirow{5}{*}{0,009} \\
\hline & Petróleo y Energía & 35 & 58,41 & & \\
\hline & Servicios de Consumo & 10 & 34,45 & & \\
\hline & Servicios Financieros e inmobiliarios & 25 & 50,62 & & \\
\hline & Tecnología y telecomunicaciones & 10 & 82,50 & & \\
\hline
\end{tabular}


Se observa que existen discrepancias entre los seis rangos, determinando que no son iguales. Además, si se estudia detalladamente el índice se puede decir que el sector más representativo, en cuanto a un mayor nivel de divulgación de Capital Estructural, es el de Tecnología y Telecomunicaciones; siendo el que menos información suministra el sector industrial correspondiente a Servicios de Consumo. Además, la $\chi^{2}(5)$ toma un valor de 15,362 , con un $p=0,009$, con lo que se puede corroborar que existen diferencias significativas entre los sectores analizados de acuerdo con su nivel de divulgación sobre Capital Estructural.

Por tanto, se puede afirmar que existen diferencias significativas entre el nivel de divulgación de información del Capital Estructural y la pertenencia a un determinado sector.

Ahora bien, para conocer entre qué pares de sectores existen esas diferencias significativas, en la tabla VIII se muestran los resultados de la prueba post hoc.

Tabla VIII. Test de Dunn-Bonferroni

\begin{tabular}{|c|c|c|c|c|c|c|}
\hline & & \multirow{2}{*}{$\begin{array}{l}\text { Diferencia } \\
\text { de medias }\end{array}$} & \multirow{2}{*}{$\begin{array}{l}\text { Error } \\
\text { típico }\end{array}$} & \multirow{2}{*}{ Sig. } & \multicolumn{2}{|c|}{$\begin{array}{c}\text { Intervalo de confianza } \\
\text { al } 95 \%\end{array}$} \\
\hline & & & & & $\begin{array}{l}\text { Límite } \\
\text { inferior }\end{array}$ & $\begin{array}{l}\text { Límite } \\
\text { superior }\end{array}$ \\
\hline \multirow{5}{*}{ 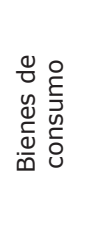 } & Industria y construcción & $-0,133$ & 0,107 & 1,000 & $-0,455$ & 0,189 \\
\hline & Petróleo y energía & $-0,071$ & 0,106 & 1,000 & $-0,390$ & 0,247 \\
\hline & Servicios de consumo & 0,083 & 0,122 & 1,000 & $-0,282$ & 0,448 \\
\hline & Servicios financieros e inmobiliarios & 0,003 & 0,109 & 1,000 & $-0,323$ & 0,330 \\
\hline & Tecnología y telecomunicaciones & $-0,250$ & 0,122 & 0,634 & $-0,615$ & 0,115 \\
\hline \multirow{5}{*}{ 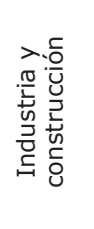 } & Bienes de consumo & 0,133 & 0,107 & 1,000 & $-0,189$ & 0,455 \\
\hline & Petróleo y energía & 0,062 & 0,055 & 1,000 & $-0,104$ & 0,228 \\
\hline & Servicios de consumo & 0,217 & 0,081 & 0,131 & $-0,027$ & 0,460 \\
\hline & Servicios financieros e inmobiliarios & 0,137 & 0,060 & 0,376 & $-0,044$ & 0,317 \\
\hline & Tecnología y telecomunicaciones & $-0,117$ & 0,081 & 1,000 & $-0,360$ & 0,127 \\
\hline \multirow{5}{*}{$\begin{array}{l}> \\
\frac{0}{d} \\
\frac{0}{0} \\
\frac{1}{0} \\
\frac{1}{4} \\
\frac{1}{2}\end{array}$} & Bienes de consumo & 0,071 & 0,106 & 1,000 & $-0,247$ & 0,390 \\
\hline & Industria y construcción & $-0,062$ & 0,055 & 1,000 & $-0,228$ & 0,104 \\
\hline & Servicios de consumo & 0,155 & 0,080 & 0,819 & $-0,084$ & 0,394 \\
\hline & Servicios financieros e inmobiliarios & 0,075 & 0,058 & 1,000 & $-0,100$ & 0,249 \\
\hline & Tecnología y telecomunicaciones & $-0,179$ & 0,080 & 0,405 & $-0,418$ & 0,060 \\
\hline \multirow{5}{*}{ 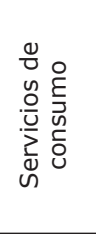 } & Bienes de consumo & $-0,083$ & 0,122 & 1,000 & $-0,448$ & 0,282 \\
\hline & Industria y construcción & $-0,217$ & 0,081 & 0,131 & $-0,460$ & 0,027 \\
\hline & Petróleo y energía & $-0,155$ & 0,080 & 0,819 & $-0,394$ & 0,084 \\
\hline & Servicios financieros e inmobiliarios & $-0,080$ & 0,083 & 1,000 & $-0,329$ & 0,169 \\
\hline & Tecnología y telecomunicaciones & $-0,333$ & 0,099 & 0,016 & $-0,631$ & $-0,035$ \\
\hline \multirow{5}{*}{ 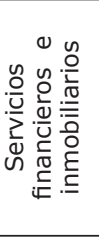 } & Bienes de consumo & $-0,003$ & 0,109 & 1,000 & $-0,330$ & 0,323 \\
\hline & Industria y construcción & $-0,137$ & 0,060 & 0,376 & $-0,317$ & 0,044 \\
\hline & Petróleo y energía & $-0,075$ & 0,058 & 1,000 & $-0,249$ & 0,100 \\
\hline & Servicios de consumo & 0,080 & 0,083 & 1,000 & $-0,169$ & 0,329 \\
\hline & Tecnología y telecomunicaciones & $-0,253$ & 0,083 & 0,043 & $-0,503$ & $-0,004$ \\
\hline \multirow{5}{*}{ 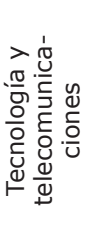 } & Bienes de consumo & 0,250 & 0,122 & 0,634 & $-0,115$ & 0,615 \\
\hline & Industria y construcción & 0,117 & 0,081 & 1,000 & $-0,127$ & 0,360 \\
\hline & Petróleo y energía & 0,179 & 0,080 & 0,405 & $-0,060$ & 0,418 \\
\hline & Servicios de consumo & 0,333 & 0,099 & 0,016 & 0,035 & 0,631 \\
\hline & Servicios financieros e inmobiliarios & 0,253 & 0,083 & 0,043 & 0,004 & 0,503 \\
\hline
\end{tabular}


Los resultados sugieren que hay una diferencia significativa, $p$-valor $0,016<0,05$, entre los sectores de Tecnología y Telecomunicaciones y el de Servicios de Consumo, respecto al suministro de información relativa al Capital Estructural. De la misma manera, existen diferencias significativas entre el sector de Tecnologías y Telecomunicaciones y el sector Financiero e Inmobiliario, $p$-valor $0,043<0,05$. Dichos resultados son coherentes con los resultados anteriormente obtenidos en el test de Kruskall-Wallis.

Así, se corrobora que el sector de Tecnologías y Telecomunicaciones es el más intensivo en la generación de Capital Estructural, al ser de alta tecnología $y$, por tanto, intensivo en intangibles, en I+D. En cuanto al menos intensivo en conocimiento organizacional, y por tanto, el que menos información suministra al respecto, es el sector de Servicios de Consumo y el de Servicios Financieros e Inmobiliarios, al ser sectores con intensidad leve en I+D. Lo cual coincide con la clasificación establecida por la OECD (2001) de las actividades basadas en el conocimiento.

\section{CONCLUSIONES}

A pesar de la importancia que tiene para las empresas suministrar información del Capital Estructural, puesto que puede suponer una mejora de su imagen y reputación para todos los stakeholders interesados en ella, y por lo tanto, un incremento de la confianza depositada en las mismas, este estudio empírico ha evidenciado que es escasa la cantidad de información que se suministra respecto al conocimiento organizacional. Quizás ello se debe al miedo que pueden tener algunas empresas a revelar información que pueda ser útil para sus competidores, pudiendo dañar la posición de liderazgo de la empresa por el hecho de poseer ciertos intangibles que son las fuentes de ventaja competitiva a largo plazo.

Sin embargo, conviene destacar que la mayoría de las empresas están suministrando de manera voluntaria información referente a su filosofía y cultura, enviando señales a sus grupos de interés respecto al buen quehacer de la empresa, es decir, informando de su manera y forma de actuar, asegurando con ello su contribución a la creación de valor de estos grupos.

Además, ha quedado demostrado que las empresas, a lo largo de los años objeto de estudio, han experimentado escasos cambios en los niveles de información suministrados, no siendo significativas las modificaciones producidas en los mismos, las empresas mantienen a lo largo del tiempo la cantidad de información que suministran sobre su Capital Estructural, a pesar de encontrarse inmersas en la Era del Conocimiento.

Por otro lado, aquellos sectores más intensos en conocimiento son los que suministran mayor cantidad de información respecto a su Capital Estructu- ral, siendo las empresas del sector de las Tecnologías y Telecomunicaciones las que más información divulgan. Ello debido, principalmente, a que son sectores que han invertido mucho en intangibles, en concreto, en nuevas tecnologías y en I+D y, por tanto, quieren suministrar información al exterior de la inversión que han realizado y que generará riqueza para las mismas a largo plazo. Cuestión lógica, ya que, para estas empresas su Capital Estructural es primordial desde la perspectiva de la innovación. Si bien, el sector de Bienes de Consumo es el que menor información suministra, ello puede deberse al hecho de ser poco intensivo en conocimiento, en intangibles, y porque no desean suministrar más información de la exigida legalmente (voluntaria) por miedo a una regulación más restrictiva en dicho sector.

Con este trabajo, se ha pretendido contribuir a la literatura sobre el suministro de información voluntaria sobre el Capital Estructural en España.

No obstante, este trabajo presenta varias limitaciones. En primer lugar, la fuente de obtención de los datos se ha limitado exclusivamente a los informes anuales, sin embargo en futuros estudios sería conveniente el análisis de otros medios de comunicación que puede utilizar la empresa, por ejemplo notas de prensa, informes de responsabilidad social corporativa, etc. En segundo lugar, la muestra no es representativa de la población total de empresas españolas, ya que el estudio se ha centrado en las mejores empresas, aquellas pertenecientes al IBEX 35. Y, en tercer lugar, en la construcción del índice no se ha realizado ninguna distinción en el carácter de la información divulgada, siendo interesante para próximos trabajos distinguir entre información de carácter cuantitativo y cualitativo.

Por último, señalar que sería conveniente como una futura línea de investigación, el estudio de aquellos factores que hacen más proclives a las empresas a llevar a cabo una estrategia de comunicación de su Capital Estructural, al igual que un estudio comparativo de los años 2008 a 2012.

\section{BIBLIOGRAFÍA}

Abeysekera, I. (2007). Intellectual capital reporting between a developing and developed nation. Journal of Intellectual Capital, vol. 8 (2), 329-345. http://dx.doi.org/10.1108/14691930710742871

Abeysekera, I.; Guthrie, J. (2005). An empirical investigation of annual reporting trends of intellectual capital in Sri Lanka. Critical Perspectives on Accounting, vol. 16 (3), 151-163. http://dx.doi. org/10.1016/S1045-2354(03)00059-5

Acerinox (2007). Informe Annual. http://www. acerinox.com/opencms803/export/sites/acerinox/ Galeria_documentos/Informe_Anual_2007.pdf [Consulta: 19-2-2014]

Acciona (2008). Informe Annual. http://memoria2008. acciona.es/media/33473/informe anual completo(pdf).pdf [Consulta: 19-4-2013] 
April, K. A.; Bosma, P.; Deglon, D. A. (2003). IC measurement and reporting: Establishing a practice in SA mining. Journal of Intellectual Capital, vol. 4 (2), 165-180. http://dx.doi. org/10.1108/14691930310472794

Beattie, V.; Thomson, S. J. (2007). Lifting the lid on the use of content analysis to investigate intellectual capital disclosures. Accounting Forum, vol. 31 (2), 129-163. http://dx.doi.org/10.1016/j. accfor.2007.02.001

Bontis, N. (2001). Assessing knowledge assets: a review of the models used to measure intellectual capital. International Journal of Management Reviews, vol. 3 (1), 41-60. http://dx.doi. org/10.1111/1468-2370.00053

Bozzolan, S.; Favotto, F.; Ricceri, F. (2003). Italian annual intellectual capital disclosure: An empirical analysis. Journal of Intellectual Capital, vol. 4 (4), 543-558. http://dx.doi. org/10.1108/14691930310504554

Brennan, N. (2001). Reporting intellectual capital in annual reports: Evidence from Ireland. Accounting, Auditing \& Accountability Journal, vol. 14 (4), 423-436. http://dx.doi. org/10.1108/09513570110403443

Brooking, A. (1997). El capital intelectual: el principal activo de las empresas del tercer milenio. Barcelona; Paidós Empresa.

Bueno Campos, E. (1998). El capital intangible como clave estratégica en la competencia actual. Boletín de Estudios Económicos, vol. 53 (164), 207-229.

Carrillo-Durán, M. V.; Nuño-Moral, M. V. (2010). La documentación en la evaluación y gestión de la imagen corporativa. El profesional de la información, vol. 19 (2), 123-132. http://dx.doi. org/10.3145/epi.2010.mar.02

Chaín Navarro, C. (2005). Propuesta de un modelo bidimensional de análisis de contenido de los sistemas webs de las administraciones locales españolas. Revista Española de Documentación Científica, vol. 28 (3), 309-333.

Davenport, T. H.; Prusak, L. (1998). Working Knowledge: How Organizations Manage What They Know. Cambridge; Harvard Business School Press.

David, P.; Foray, D. (2002). Fundamentos economicos de la sociedad del conocimiento. Comercio Exterior, vol. 52 (6), 472-490.

Drucker, P. F. (1993). La sociedad poscapitalista. Barcelona; Apóstrofe.

Ensslin, S. R.; De Carvalho, F. N. (2007). Voluntary disclosure of intellectual capital in the Brazilian context: an investigation informed by the international context. International Journal of Accounting, Auditing and Performance Evaluation, vol. 4 (4-5), 478-500. http://dx.doi.org/10.1504/ IJAAPE. 2007.017089

Euroforum. (1998). Medición del capital intelectual: Modelo intelect. Madrid; Instituto Universitario Euroforum Escorial.

Ferran-Ferrer, N.; Pérez-Montoro, M. (2009). Gestión de la información personal en usuarios avanzados en TIC. El profesional de la información, vol. 18 (4), 365-373. http://dx.doi.org/10.3145/epi.2009. jul.02

Ferrer, A. (2008). Información en la empresa para innovar y competir. El profesional de la información, 17 (5), 481-486. http://dx.doi.org/10.3145/ epi.2008.sep.01

Gan, K.; Saleh, Z.; Abessi, M. (2008). Corporate Governance, Ownership Structures and Intellectual Capital Disclosures: Malaysian Evidence. Paper presentado al 16th Conference on the Theories and Practices of Securities and Finance Markets. Department of Finance, National Sun Yat-Sen University. 5-6 December 2008, Kaohsiung, Taiwan.

García-Meca, E.; Martínez, I. (2005). Assessing the quality of disclosure on intangibles in the Spanish capital market. European Business Review, vol. 17 (4), 305-313. http://dx.doi. org/10.1108/09555340510607352

Goh, P. C.; Lim, K. P. (2004). Disclosing intellectual capital in company annual reports: Evidence from Malaysia. Journal of Intellectual Capital, vol. 5 (3), 500-510. http://dx.doi. org/10.1108/14691930410550426

Grant, R. M. (1996). Toward a knowledge-based theory of the firm. Strategic Management Journal, vol. 17 (10), 109-122.

Guthrie, J.; Petty, R. (2000). Intellectual capital: Australian annual reporting practices. Journal of Intellectual Capital, vol. 1 (3), 241-251. http:// dx.doi.org/10.1108/14691930010350800

Guthrie, J.; Petty, R.; Ricceri, F. (2006). The voluntary reporting of intellectual capital: Comparing evidence from Hong Kong and Australia. Journal of Intellectual Capital, vol. 7 (2), 254-271. http:// dx.doi.org/10.1108/14691930610661890

Guthrie, J.; Petty, R.; Yongvanich, K.; Ricceri, F. (2004). Using content analysis as a research method to inquire into intellectual capital reporting. Journal of Intellectual Capital, vol. 5 (2), 282-293. http://dx.doi.org/10.1108/14691930410533704

Hernández, R.; Fernández, C.; Baptista, P. (2006). Metodología de la Investigación. México; McGrawHill Interamericana.

Kaplan, R. S.; Norton, D. P. (1992). The balanced scorecard-measures that drive performance. Harvard Business Review, vol. 70 (1), 71-79.

Krippendorff, K. (2004). Content analysis: An introduction to its methodology. California; Sage Publications, Inc.

Lascurain, M.L.; Madera-Jaramillo, M.J.; Ortoll, E.; Sanz, E. (2010): Capacidad innovadora de la Comunidad de Madrid a partir de las patentes concedidas entre 1996 y 2007. Revista Española de Documentación Científica, vol. 33 (3), 458-479. http://dx.doi.org/10.3989/redc.2010.3.761

Ley $26 / 2003$, de 17 de julio, sobre transparencia de las sociedades anónimas cotizadas (BOE No. 171, 18 de julio 2003).

Li, J.; Pike, R.; Haniffa, R. (2008). Intellectual capital disclosure and corporate governance structure in 
UK firms. Accounting and Business Research, vol. 38 (2), 137-159. http://dx.doi.org/10.1080/00014 788.2008 .9663326

Nonaka, I.; Takeuchi, H. (1995). The knowledgecreating company, New York; Oxford University Press.

OECD, Organisation for Economic Co-operation and Development. (1996). The Knowledge-based economy. Paris; Organisation for Economic Cooperation and Development.

OECD, Organisation for Economic Co-operation and Development. (2001). OECD Science, Technology and Industry Scoreboard 2001: Towards a Knowledge-based Economy. Paris; OECD Publishing.

Oliveras, E.; Gowthorpe, C.; Kasperskaya, Y.; Perramon, J. (2008). Reporting intellectual capital in Spain. Corporate Communications: An International Journal, vol. 13 (2), 168-181. http:// dx.doi.org/10.1108/13563280810869596

Orden del Ministerio de Economía 3722/2003, de 26 de diciembre, sobre el informe anual de gobierno corporativo y otros instrumentos de información de las sociedades anónimas cotizadas y otras entidades (BOE No. 7, 8 de enero 2004)

Ortiz de Urbina, M. (2003). Medición y auditoría del capital intelectual. El profesional de la información, vol. 12 (4), 282-289. http://dx.doi.org/10.1076/ epri.12.4.282.16901

Ramos Vielba, I.; Clabo Clemente, N. (2008). Calidad de las sedes web de las OTRI universitarias andaluzas: contenidos, usabilidad y accesibilidad. Revista Española de Documentación Científica, vol. 31 (3), 366-395.

Sancho, R. (2007). Innovación industrial. Revista Española de Documentación Científica, vol. 30 (4), 553-564.

Steenkamp, N. (2007). Intellectual capital reporting in New Zealand: refining content analysis as a research method. Auckland University of Technology, Auckland (New Zealand). Tesis Doctoral.

Sveiby, K. E. (1997). The New Organizational Wealth: Managing y Measuring Knowledge-based Assets. San Francisco; Berrett-Koehler Publishers, p. 9.

Vickery, G. (1999). La medición de la economía del conocimiento: medición y presentación de los intangibles. Ekonomiaz, vol. 3 (45), 160-185.

Vandemaele, S. N.; Vergauwen, P. G. M. C.; Smits, A. J. (2005). Intellectual capital disclosure in The Netherlands, Sweden and the UK: A longitudinal and comparative study. Journal of Intellectual Capital, vol. 6 (3), 417-426. http://dx.doi. org/10.1108/14691930510611148 